\section{Desenvolvimento de geopolímeros de baixa condutividade térmica}

\author{
Development of geopolymers of \\ low thermal conductivity
}

Vanderlei Felizardo ${ }^{1}$, Vitor de Souza Nandi ${ }^{1}$, Karina Donadel ${ }^{1}$ Rui Novais ${ }^{2}$, João Labrincha ${ }^{2}$, Alexandre Zaccaron ${ }^{3}$

\author{
${ }^{1}$ Centro Universitário Barriga Verde - UNIBAVE, 88845-000, Cocal do Sul-SC, Brasil. \\ e-mail: felizardo.vv@hotmail.com; vitorsnprof@gmail.com; karina.donadel@unibave.net \\ ${ }^{2}$ Departamento de Engenharia de Materiais e Cerâmica, Universidade de Aveiro, 3810-193, Aveiro, Portugal. \\ e-mail: ruimnovais@ua.pt; jal@ua.pt \\ ${ }^{3}$ Laboratório Técnico de Cerâmica Vermelha - LABCER, 88830-000, Morro da Fumaça, SC, Brasil \\ e-mail: alexandrezaccaron@hotmail.com
}

\section{RESUMO}

Os materiais de isolamento térmico convencionais, tais como a lã de vidro e o poliestireno extrudado, possuem importantes limitações técnicas. Os geopolímeros emergem como uma alternativa devido às suas excelentes propriedades. Neste trabalho, teve-se por objetivo descrever o desenvolvimento de geopolímeros de baixa condutividade térmica. Na produção dos geopolímeros utilizou-se metacaulim $\left(\mathrm{Al}_{2} \mathrm{O}_{3} \cdot 2 \mathrm{SiO}_{2}\right)$ que, devido à sua elevada reatividade, permite produzir polímeros inorgânicos, com propriedades físicas interessantes para aplicações em isolação térmica. Amostras de aluminossilicato foram ativadas com hidróxido de sódio (Na$\mathrm{OH})$ e silicato de sódio $\left(\mathrm{Na}_{2} \mathrm{SiO}_{3}\right)$. Amostras de geopolímeros leves foram produzidas com adição de diferentes percentagens de um formador de poros $(0,5,1,2,3,4 \%)$. As amostras produzidas foram caracterizadas quanto à: resistência, compressão, absorção de água, condutividade térmica, densidade aparente e microestrutura.

Palavras-chave: Isolamento térmico; Geopolímeros; Condutividade térmica.

\begin{abstract}
Conventional thermal insulation materials, such as glass wool and extruded polystyrene, owned-in significant technical limitations. The geopolymers emerge as an alternative due to their properties. In this sense the objective of this work is to describe the develop geopolymers with low thermal conductivity. Samples of geopolymers were produced from metakaolin $\left(\mathrm{Al}_{2} \mathrm{O}_{3} \cdot 2 \mathrm{SiO}_{2}\right)$ which, due to its high reactivity, made it possible to produce inorganic polymers with interesting physical properties, which can be used for thermal insulation. The samples of aluminosilicate were activated with sodium hydroxide $(\mathrm{NaOH})$ and sodium silicate $\left(\mathrm{Na}_{2} \mathrm{SiO}_{3}\right)$. Samples of light geopolymers were produced with addition of different percentages of a pore former $(0.5,1$, 2, 3, 4\%). The samples were characterized with respect to: compressive strength, water absorption, thermal conductivity, density and microstructure.
\end{abstract}

Keywords: Thermal insulation; Geopolymers; Thermal conductivity.

\section{INTRODUÇÃO}

O termo geopolímero foi introduzido por Davidovits para representar polímeros inorgânicos [1]. A geopolimerização ou ativação alcalina consiste na reação, em meio altamente alcalino, entre materiais ricos em sílica e em alumina [2]. Nesta reação são criadas ligações do tipo Si-O-Al-O [2]. Dada a necessidade de assegurar um elevado grau de amorfismo estrutural, a matéria-prima mais utilizada como aluminossilicato, é o metacaulim. Este produto resulta da calcinação a temperaturas moderadas $\left(700\right.$ a $\left.800^{\circ} \mathrm{C}\right)$ do caulim comum [3]. A geopolimerização requer a utilização de ativadores alcalinos, para tanto, utiliza-se uma mistura de $\mathrm{NaOH}$ e $\mathrm{Na}_{2} \mathrm{SiO}_{3}$ [4]. Nos últimos anos, essa tecnologia tem se revelado-uma alternativa aos sistemas ligantes comuns que utilizam cimento Portland [5]. Contudo, estudos que consideram sua utilização como material de isola- 
mento térmico são escassos [6, 7]. Devido às qualidades (elevada estabilidade térmica, estrutura extremamente porosa, não inflamáveis, produzidos por meio de tecnologia de baixo custo e de baixo impacto ambiental) os geopolímeros proporcionam a possibilidade de utilização como material para isolação térmica.

Neste trabalho foram produzidas amostras de geopolímeros de baixa condutividade térmica produzidas por meio de formulações com diferentes percentagens de formador de poros [8]. A influência da percentagem de porogêneo nas propriedades das amostras foram avaliadas, bem como as propriedades de resistência mecânica, absorção de água e condutividade térmica. O nível de porosidade foi avaliado por microscopia óptica e eletrônica de varredura (MEV).

\section{MATERIAIS E MÉTODOS}

Para produção das amostras, foi utilizado um metacaulim comercial (Argirec B24, Univar B. V) e um ativador alcalino composto de hidróxido de sódio $(\mathrm{NaOH})$, fornecido pela Sigma-Aldrich, e silicato de sódio $\left(\mathrm{Na}_{2} \mathrm{SiO}_{3}\right)$. Como formador de poros utilizou-se o peróxido de hidrogênio $\left(\mathrm{H}_{2} \mathrm{O}_{2}\right) 10$ volumes.

A análise granulométrica foi efetuada por sedimentação e interferência a laser, utilizando o equipamento Coulter LS230, que permite a medição de partículas com diâmetro variando entre 0,04 $\mu$ m e $2000 \mu \mathrm{m}$ [9]. A determinação da composição química do metacaulim foi efetuada recorrendo-se à fluorescência de raios $X$, baseada na medição da intensidade dos raios $X$ [10] emitidos pelos elementos que constituem a amostra. Para tanto, utilizou-se o equipamento Philips X', Pert PRO MPD [11].

A identificação das fases cristalinas do metacaulim foi efetuada por difratometria de raios X (DRX). As análises foram realizadas no equipamento Rigaku Geigerflex D/max-Series, empregando-se uma velocidade de varredura de $3^{\circ} 2 \theta / \mathrm{min}$ para o intervalo entre 10 e $80^{\circ}(2 \theta)$

A morfologia das partículas de metacaulim foi avaliada por microscopia eletrônica de varredura (MEV). Nessa análise utilizou-se o microscópio Hitachi S4100, equipado com um sistema de espectroscopia de energia dispersiva (EDS), capaz de identificar os elementos químicos presentes.

A formulação de referência foi preparada considerando-se as seguintes percentagens entre as matériasprimas: 34,29\% de metacaulim, 45,71\% de silicato de sódio e 20\% de hidróxido de sódio. Na primeira etapa das preparação amostras, efetuou-se a mistura entre os ativadores $\left(\mathrm{NaOH}\right.$ e $\left.\mathrm{Na}_{2} \mathrm{SiO}_{3}\right)$ durante 5 minutos em um misturador intensivo (KITCHENAID KEA30 - STAND MIXER - 220 V). Em seguida, adicionou-se ao metacaulim o ativador composto (previamente homogeneizado) e realizou-se a agitação da mistura durante 10 min. Em seguida, a mistura foi vertida para moldes plásticos cilíndricos (2,21 cm de diâmetro e 4,44 cm de altura) vedados com película aderente de modo a evitar as trocas de umidade com o meio exterior. As amostras assim preparadas foram colocados numa câmara climática (climatic chamber Fitoclima 300EP10, Aralab) de modo a promover o endurecimento do material. A temperatura e a umidade relativa selecionadas para a cura foram, respectivamente, $40^{\circ} \mathrm{C}$ e $60 \%$.

Após 24 horas as amostras foram desmoldadas, tendo permanecido na câmara climática até perfazer sete dias de cura. No final deste período, as amostras foram retiradas da câmara climática tendo a cura decorrido à temperatura e umidade ambientes.

A produção de amostras porosas envolveu uma etapa adicional, na qual o formador de poros foi adicionado à mistura metacaulim e ativador composto, sendo o tempo de mistura de 2 min. Foram preparadas formulações contendo 0,5, 1, 2, 3 e 4\% (em massa) de $\mathrm{H}_{2} \mathrm{O}_{2}$.

A condutividade térmica das amostras foi medida à temperatura ambiente, utilizando-se o equipamento KD2 ProTermal Properties Analyzer, após calibração do mesmo com um padrão (DB1175). Para minimizar o erro associado às medições, foram efetuadas, no mínimo, 5 medidas para cada amostra.

Os ensaios de compressão das amostras foram realizados numa máquina universal de ensaios mecânicos (Shimadzzu - Autograph AG-25 TA), mantendo-se um velocidade de aplicação da carga de 0,5 mm/min, até à ruptura do material.

A morfologia das amostras processadas foi avaliada utilizando-se um microscópio óptico (Leica E4HD). Para essa análise as amostras foram cortadas com uma máquina de corte (Struers Secotom-10), mantendo-se as superfícies planas, de modo a permitir visualizar os poros.

A avaliação microestrutural dos geopolímeros foi efetuada por MEV, tendo a identificação dos elementos químicos presentes sido efetuada por EDS.

A absorção de água $(A A)$ das amostras produzidas foi avaliada determinando inicialmente a massa seca $\left(m_{\text {seca }}\right)$, onde cada amostra foi mantida em estufa por 24 horas à temperatura de $40^{\circ} \mathrm{C}$, sendo posteriormente imersas em água durante $24 \mathrm{~h}$ ou até não se verificar variação da massa. O excesso de água superficial foi 
removido determinando-se a $m_{\text {saturada }}$ das amostras. A absorção de água das amostras foi determinada por meio da seguinte equação (01):

$$
A A(\%)=\frac{m_{\text {saturada }}-m_{\text {seca }}}{m_{\text {seca }}} \times 100
$$

A partir da determinação do volume e da massa das amostras, foram obtidas suas densidades aparentes $(\rho)$, de acordo com a seguinte equação (02):

$$
\rho=\frac{m}{v}
$$

sendo $m$ a massa (g) e $v$ o volume $\left(\mathrm{cm}^{3}\right)$.

\section{RESULTADOS E DISCUSSÃO}

Na figura 1 está evidente a distribuição granulométrica do metacaulim. Observa-se que suas partículas apresentam uma dimensão média de $5 \mu \mathrm{m}$.

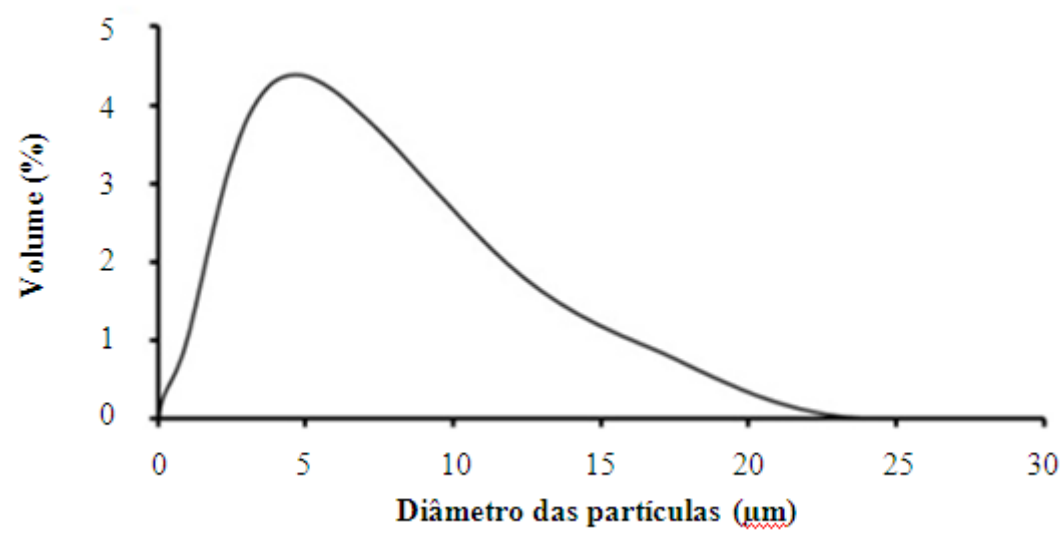

Figura 1: Distribuição granulométrica do metacaulim.

Na figura 2 apresenta-se a micrografia obtida por MEV do metacaulim utilizado. Como se pode verificar, as partículas apresentam morfologia irregular e dimensões inferiores a $20 \mu \mathrm{m}$. A partir das análises efetuadas por EDS (figura 3) verificou-se que o metacaulim é constituído pelos elementos silício e alumínio.

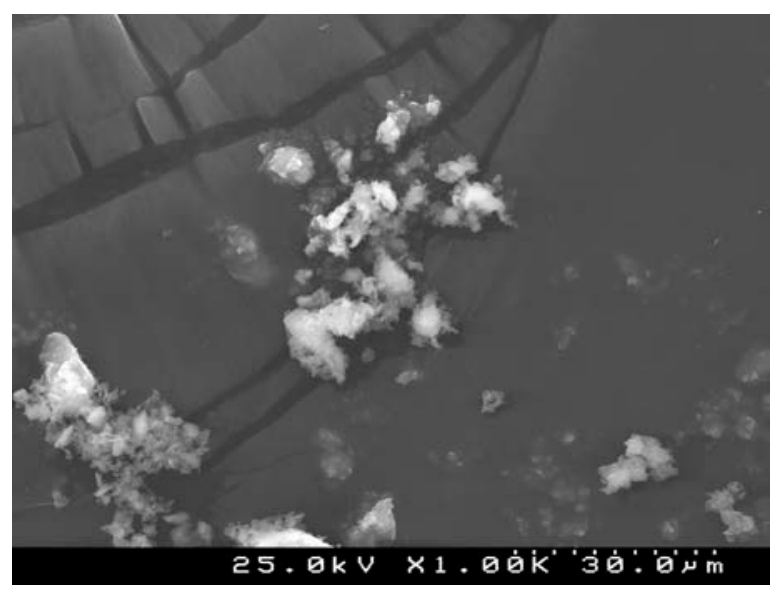

Figura 2: Micrografia do metacaulim obtida por MEV. 


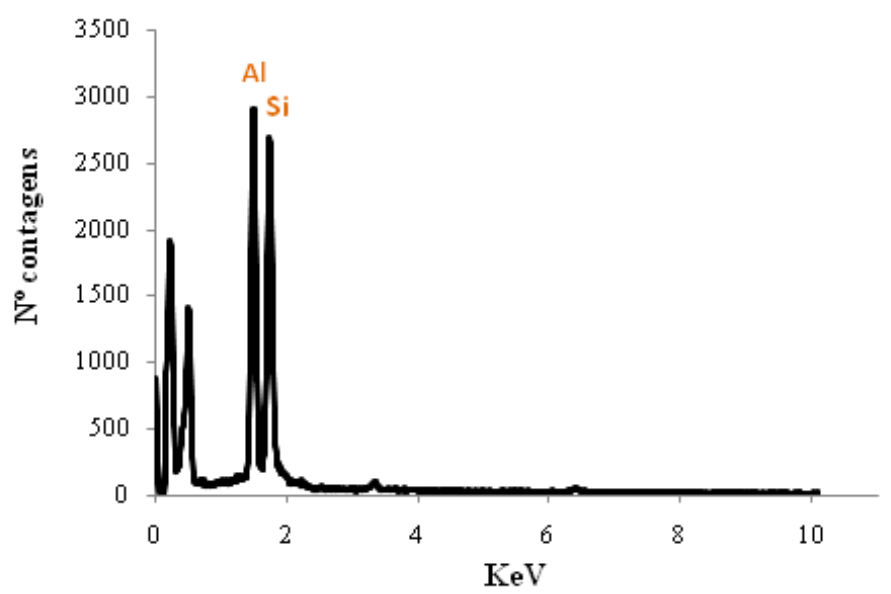

Figura 3: Análise química elementar do metacaulim obtida por EDS.

A composição química do MK, determinada por FRX, mostrou que seus componentes majoritários são sílica $\left(\mathrm{SiO}_{2}\right)$ e alumina $\left(\mathrm{Al}_{2} \mathrm{O}_{3}\right)$, o que está de acordo com os resultados obtidos por EDS (Figura 3).

Tabela 1: Composição química do metacaulim obtida por FRX.

\begin{tabular}{c|c}
\hline Óxidos & $\begin{array}{c}\text { MK } \\
\text { (\% em massa) }\end{array}$ \\
\hline $\mathrm{SiO}_{2}$ & 54,4 \\
\hline $\mathrm{TiO}_{2}$ & 1,55 \\
\hline $\mathrm{Al}_{2} \mathrm{O}_{3}$ & 39,4 \\
\hline $\mathrm{Fe}_{2} \mathrm{O}_{3}$ & 1,75 \\
\hline $\mathrm{MgO}$ & 0,14 \\
\hline $\mathrm{CaO}$ & 0,10 \\
\hline $\mathrm{MnO}$ & 0,01 \\
\hline $\mathrm{K}_{2} \mathrm{O}$ & 1,03 \\
\hline $\mathrm{P}_{2} \mathrm{O}_{5}$ & 0,06 \\
\hline $\mathrm{P} . \mathrm{F}$. & 2,66 \\
\hline
\end{tabular}

P.F. - perda ao fogo.

No difratograma do metacaulim (figura 4) observa-se que este material é altamente amorfo, contendo ainda assim elementos cristalinos (quartzo e muscovita). 


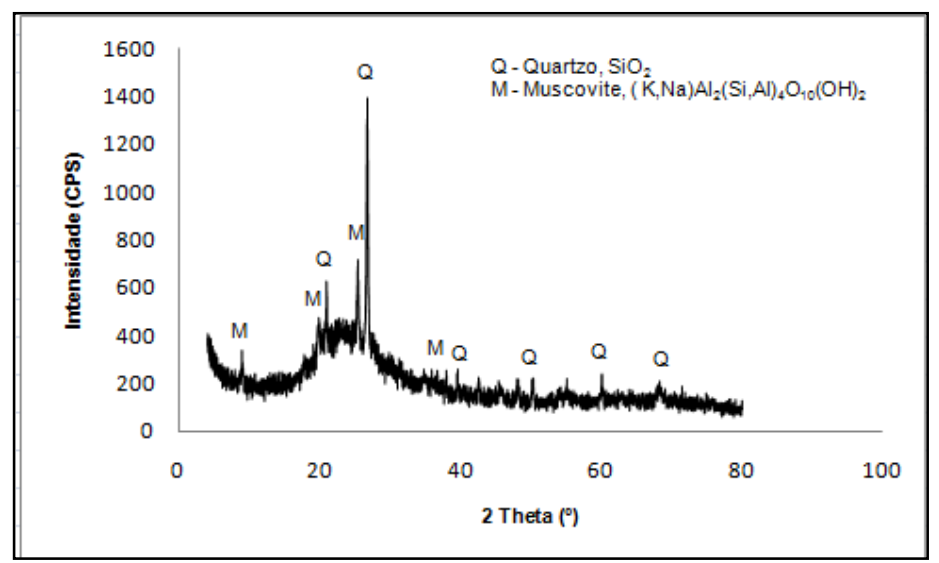

Figura 4: Difratograma do metacaulim

Na figura 5 apresentam-se micrografias ópticas dos geopolímeros, contendo diferentes percentagens de incorporação de $\mathrm{H}_{2} \mathrm{O}_{2}$, após 7 dias de cura.

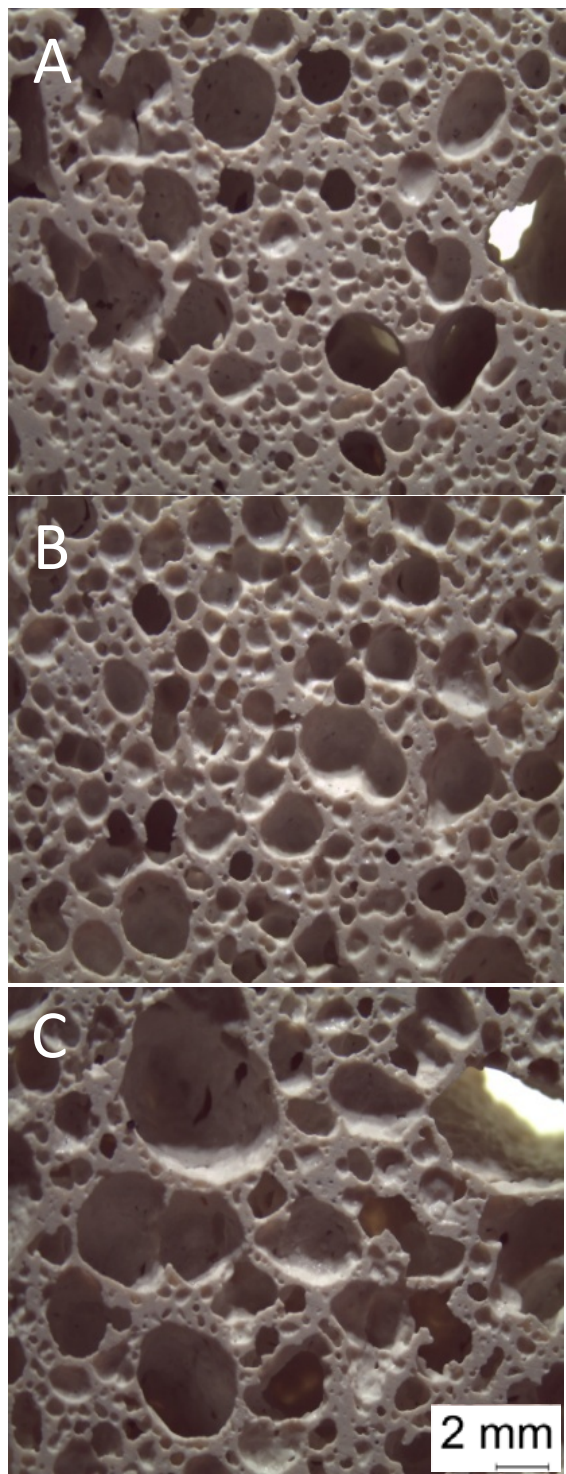

Figura 5: Micrografias de geopolímeros contendo: A) 1\%, B) 2\% e C) 3\% de $\mathrm{H}_{2} \mathrm{O}_{2}$. 
Pode-se observar que o aumento da percentagem de $\mathrm{H}_{2} \mathrm{O}_{2}$ fez aumentar o diâmetro dos poros produzidos.

Na figura 6 apresentam-se micrografias obtidas por MEV das amostras produzidas com diferente incorporação de formador de poros. Constatou-se que o número e o volume de poros produzidos foi significativamente influenciado pela percentagem de incorporação de formador de poros.
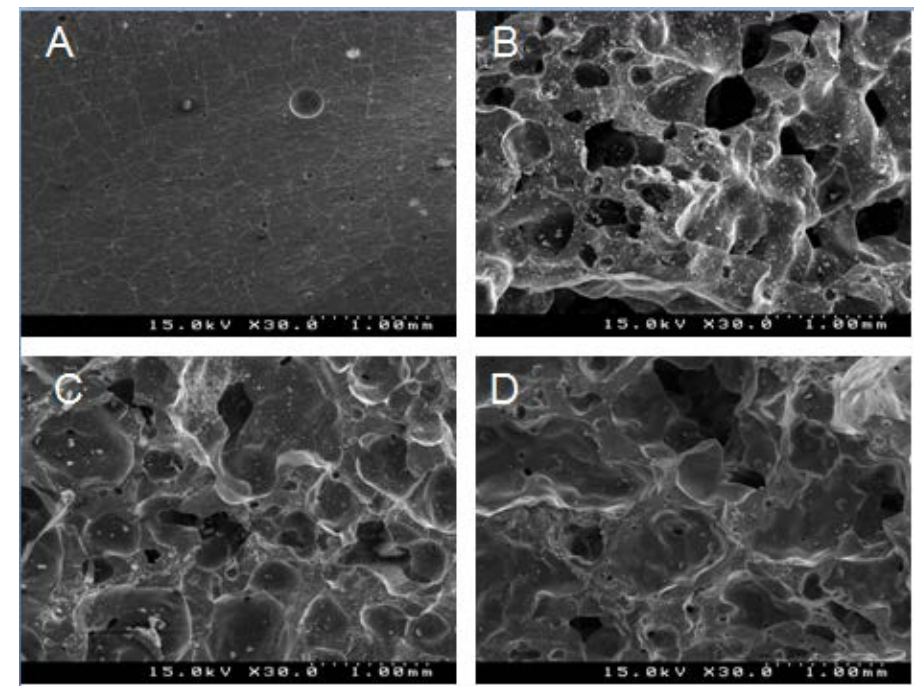

Figura 6: Micrografias obtidas por MEV dos geopolímeros produzidos: A) Padrão, B) 1\%, C) 2\% e D) 3\% de $\mathrm{H}_{2} \mathrm{O}_{2}$.

Com base nos resultados, constatou-se que a microestrutura dos geopolímeros foi significativamente modificada pela incorporação do porogêneo (agente formador de poros), comparativamente com a formulação de referência. A área dos poros aumenta com o aumento de percentagem de $\mathrm{H}_{2} \mathrm{O}_{2}$. Uma análise mais detalhada da figura indicou a presença de fissuras na microestrutura da amostra. Salienta-se que nesta análise foram utilizadas amostras que tinham sido submetidas a ensaios de resistência à compressão, o que pode explicar a presença das fissuras.

Na figura 7 apresenta-se o espectro obtido por EDS de uma amostra contendo 1\% de formador de poros. Como era de se esperar, foram detectados os elementos $\mathrm{Si}, \mathrm{Al}$, Na e O.

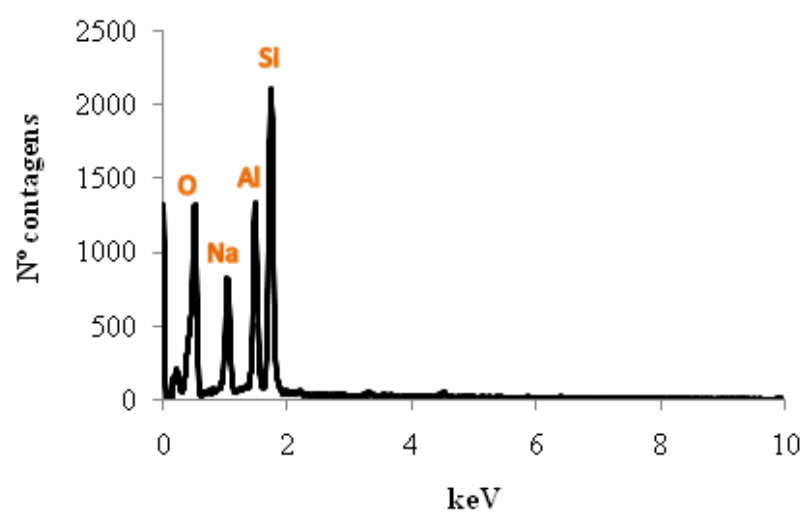

Figura 7: Análise química elementar do geopolímero com $1 \%$ de $\mathrm{H}_{2} \mathrm{O}_{2}$, obtida por EDS.

Nas figuras (8, 9, 10 e 11) observam-se a influência da percentagem de $\mathrm{H}_{2} \mathrm{O}_{2}$ na resistência à compressão, na absorção de água, na condutividade térmica e na densidade aparente das amostras. 


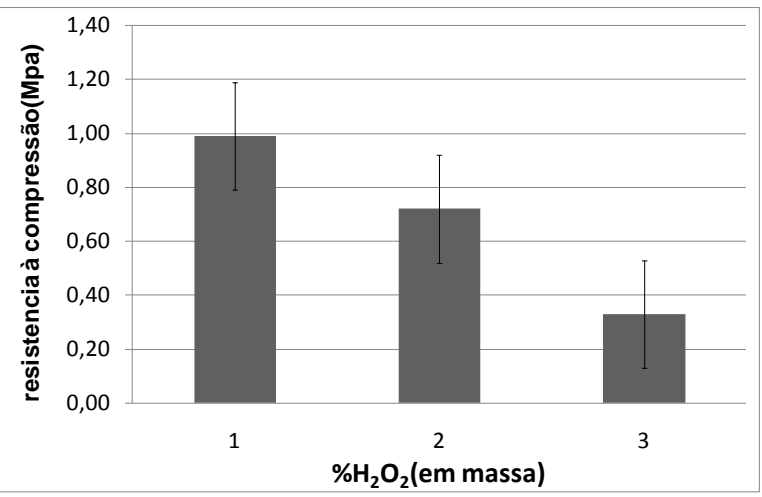

Figura 8: Influência da quantidade de $\mathrm{H}_{2} \mathrm{O}_{2}$ na resistência à compressão dos geopolímeros.

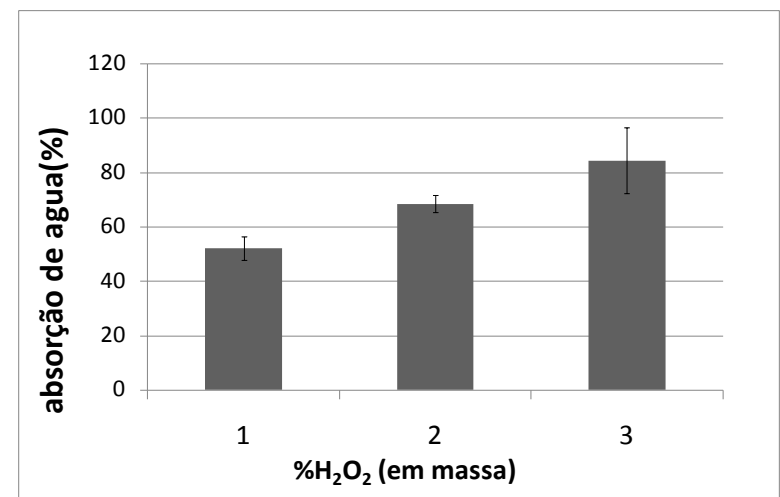

Figura 9: Influência da quantidade de $\mathrm{H}_{2} \mathrm{O}_{2}$ na percentagem de absorção de água dos geopolímeros.

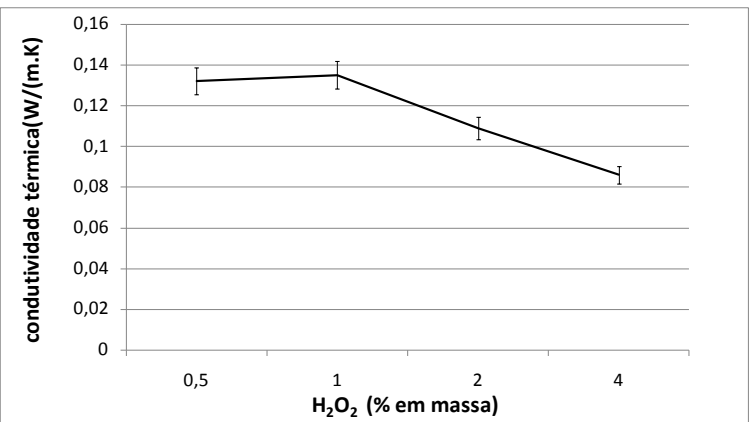

Figura 10: Influência da quantidade de $\mathrm{H}_{2} \mathrm{O}_{2}$ na condutividade térmica dos geopolímeros.

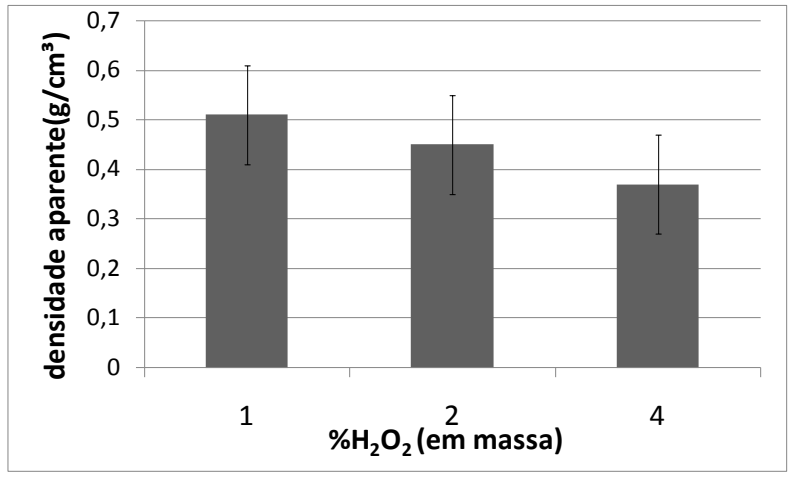

Figura 11: Influência da quantidade de $\mathrm{H}_{2} \mathrm{O}_{2}$ na densidade aparente dos geopolímeros. 
Com base nos resultados obtidos e nas análises realizadas, pode-se afirmar que a incorporação de $\mathrm{H}_{2} \mathrm{O}_{2}$ reduziu a resistência à compressão e a densidade aparente das amostras, aumentando a percentagem de absorção de água. Tal como se demonstrou anteriormente, o aumento da percentagem de formador de poros promoveu um incremento do número e do diâmetro dos poros produzidos, fato que fragilizou os geopolímeros.

Constatou-se que A condutividade térmica tendeu a diminuir com o aumento da porosidade. A maior quantidade de vazios reduziu a condutividade térmica devido à menor condutividade do ar incorporado às amostras, comparativamente à mesma propriedade do material cerâmico. Como pode ser verificado, foi possível produzir geopolímeros com valores de condutividade térmica inferiores a 0,09 W/m.K, evidenciando a possibilidade de aplicação da técnica para produção de geopolímeros para fins de isolação térmica.

\section{CONCLUSÕES}

Neste trabalho pretendeu-se avaliar a possibilidade de desenvolvimento de geopolímeros de baixa condutividade térmica para utilização como material de isolação térmica. Neste sentido, estudou-se a influência da percentagem de incorporação de um formador de poros nas propriedades dos geopolímeros. Considerando-se Os resultados obtidos, ficou evidente que é possível produzir geopolímeros com muito baixa condutividade térmica $(<0,09 \mathrm{~W} / \mathrm{m} \cdot \mathrm{K})$.

Também foi constatado que com o aumento da percentagem de $\mathrm{H}_{2} \mathrm{O}_{2}$ influenciou na densidade aparente, diminuindo o seu percentual de $0,51 \%$ na primeira amostra para $0,37 \%$ na segunda amostra. Essa diminuição também foi observada no teste de resistência à compressão, que diminuiu de $0,99 \%$ para $0,33 \%$. Por outro lado, a absorção de água aumentou significativamente (de 4,27\% para 11,99\%) o que se explica pelo incremento do número e da dimensão dos poros.

\section{AGRADECIMENTOS}

Os autores agradecem à Capes, ao CNPQ e aos profissionais do Departamento de Materiais e Cerâmica da Universidade de Aveiro-PT.

\section{BIBLIOGRAFIA}

[1] CENTEIO, J.C.S. Propriedades fisicas de argamassa geopolimérica de lamas residuais das minas de panasqueira. Dissertação M.Sc., Universidade da Beira Interior, Covilhã, Portugal, 2011.

[2] KOMNITSAS, K.; ZAHARAKI, D. "Geopolymerization: a review and prospects for the minerals industry” Mineral Engineering, 20, 14, pp. 1261-1277, nov. 2007.

[3] MARQUES, J.I.S.,Geopolimerização de cinzas e dregs da indústria de pasta de papel. Dissertação M.Sc., Universidade de Aveiro, Aveiro, Portugal. 2013.

[4] SAID-MANSOUR, M., KADRI, E.H., KENAI, S., et al."Influence of calcined kaolin on mortar properties”, Construction and Building Materials, v.25, n.5, pp. 2275-2282, may 2011.

[5] HAJJAJI, W., ANDREJKOVIČOVÁ, S., ZANELLI, C., et al. "Composition and technological properties of geopolymers based on metakaolin and red mud”,Materials and Design, v.52, pp. 648-654, dec. 2013.

[6] SEPÚVEDA, J. Geopolímeros. https://engenhariacivil.wordpress.com/2007/07/03/geopolimeros/ Acessado em agosto de 2015.

[7] VAOU, V., PANIAS, D. “Thermal insulating foamy geopolymers from perlite”, Minerals and Engineering, v. 23, n.14, pp. 1146-1151, nov. 2010.

[8] FENG, J., ZHANG, R., GONG, L., et al., "Development of porous fly-ash-based geopolymer with low thermal conductivity”, Materials and Design, v.65, pp. 529-533, jan. 2015.

[9] FRADE, J. R., PAIVA, A.T. Condutividade térmica e Calor Específico: Combustíveis, Energia e Ambiente Actividades de Projecto Laboratorial. Un. 2, Universidade de Aveiro, 2006.

[10] RODRIGUES, T.S., FARIA, P. Ensaios de caracterização laboratorial de solos com vista à sua utilização em arquitectura de terra crua. In: IV SIACOT - Seminário Ibero-Americano de Construção em Terra. Monsaraz, PROTERRA/CdT, out. 2005

[11] KOMNITSAS, K., DIMITRA Z."Geopolymerisation: A review and prospects for the minerals industry”, Minerals Engineering, v.20, 14, pp. 1261-1277, nov. 2007. 